\title{
Speckle reconstruction of photometric data observed with adaptive optics
}

\author{
K. G. Puschmann and M. Sailer
}

\begin{abstract}
Institut für Astrophysik, Freidrich-Hund-Platz 1, 37077 Göttingen, Germany
e-mail: [kgp;msailer]@astro.physik.uni-goettingen.de
\end{abstract}

Received 26 July 2005 / Accepted 3 April 2006

\begin{abstract}
To achieve the highest spatial resolution for ground-based observations one has to correct for degradations by the Earth's atmosphere. This can be done by on-line and post-facto techniques. Here we combine observations with Adaptive Optics (AO) and speckle reconstruction. As possible techniques we present two modified versions (methods $B$ and $C$ ) of the Göttingen speckle masking code and describe their application to observations of a solar active region obtained with the Swedish 1-m Solar Telescope (La Palma) by means of AO. By comparing the results with those obtained from the conventional method (method $A$ ), the influence of AO on the post-facto reconstruction is revealed. One has to account for the field dependence of the AO correction and the fitting errors of the calculated surface of the deformable mirror with respect to the according wave fronts. Both new approaches enhance the contrast in the lockpoint region less than the conventional method. Method $C$ uses simulated AO corrected speckle transfer functions (STFs). This method shows the most uniform image quality over the whole field of view and indicates the importance of the atmospheric model assumptions and the partial character of the WF correction by AO with appropriate WF error statistics. However, the consideration of field dependent speckle transfer functions in the classical speckle reconstruction (Method $B$ ) can serve as a first approach to avoid an intensity over-amplification around the AO-lockpoint region, although the rms of the contrast in the reconstructed images tends to rise slightly towards the outer parts of the field of view. Even the apparently small differences between the speckle transfer functions, used in the different reconstruction methods, cause the modulus of the estimated intensity differences to vary by up to $22 \%$.
\end{abstract}

Key words. Sun: photosphere - techniques: high angular resolution - techniques: image processing - instrumentation: adaptive optics

\section{Introduction}

The investigation of the solar fine structure and its dynamics requires data of highest angular resolution (better than 0.'1) which can be only achieved by online and post-facto techniques, i.e. the combination of Adaptive Optics (AO) and an image reconstruction method like speckle masking or phase diversity (Löfdahl \& Scharmer 1994; Paxman et al. 1996; van Noort et al. 2005).

During the last decade the Göttingen speckle masking code has been successfully applied to spectrometric and spectropolarimetric data in various fields of solar physics. The high spatial resolution obtained with this code was important for the detection of short-period acoustic waves (Wunnenberg et al. 2002) and inter-network magnetic fields (Domínguez Cerdeña et al. 2003a,b), for studies of an enhanced network region observed in $\mathrm{H} \alpha$ (Al et al. 2004), of the structure of polar faculae (Okunev \& Kneer 2004), of small-scale solar magnetic flux concentrations (Wiehr et al. 2004; Puschmann \& Wiehr 2006), as well as for polarimetry of the penumbrae of sunspots (Bello González et al. 2005) and of solar pores (Hirzberger et al. 2005). After the first solar Adaptive Optical system (Rimmele et al. 1998, 2000, 2003), the German Vacuum Tower Telescope (VTT) at the Observatorio del Teide (von der Lühe et al. 2003), as well as the Swedish 1-m Solar Telescope (SST) at the Observatorio Roque de los Muchachos (Scharmer et al. 2003a,b) have been equipped with $\mathrm{AO}$, which provides a real-time correction of wave front distortions induced by the Earth's atmosphere.

As the AO significantly improves the resolution of raw images, but corrects image degradations only up to a certain order of Zernike polynomials (in the case of the SST
Karhunen-Loeve functions), the diffraction-limited resolution is achieved only rarely, as it requires reasonably good atmospheric seeing conditions. By subsequent application of speckle reconstruction we reach that limit more consistently. The combination is a logical consequence, as AO increases the number of those short-exposure images during a speckle burst that contain sufficient information on the fine structure required for reconstruction. The increased probability of obtaining high quality raw data, on which all image reconstruction methods depend, results in a higher significance of the reconstructed intensities and of the positions of the smallest features present in the finally obtained images, as the signal to noise ratio of the high frequency part of the power spectra is enhanced.

The wave front compensation produced by AO, however, is never perfect and the resulting optical transfer function (OTF) has an unusual structure, compared with those from purely atmospherically influenced wave fronts. Therefore the important parameters of the $\mathrm{AO}$ system must be investigated and their effects on the short- and long-exposure images analysed. In the case of speckle reconstruction, we need to have reliable model OTFs or $S T F \mathrm{~s}$, the latter describing average short-exposure $O T F \mathrm{~s}$ valid for the image burst, to deconvolve the corresponding data. As we use the $S T F$ to devide the Fourier amplitudes of the speckle data to obtain the "true" intensities of the observed object, it is vital to have the best possible estimate for the OTFs at the time of observation. Until now, only atmospherically degraded $S T F$ s have been assumed for speckle reconstruction, calculated numerically from the theoretical integral expression based on the Korff model (Korff 1973). Here, only one atmospheric parameter was varied, 
namely the Fried parameter $r_{0}$ (Fried 1966). With the use of AO, however, the space of free parameters has been increased.

The most evident effect is the field dependence of AO compensation with respect to the lockpoint in the field of view (FOV). The light beams that enter the telescope from different positions in the source traverse the Earth's atmosphere with different angles. So, different volumes of the high-altitude turbulence influence the wave fronts, giving rise to varying aberrations across the FOV. The wave front sensor, however, can "see" only a small region of the Sun around the lockpoint, thus only the deformation corresponding to this direction can be sensed and used to calculate the corrective shape of the deformable mirror (DM). Consequently, the compensation outside this image region decreases and will even further degrade the original wave front, if the correlation of the mirror form and the wavefront drops below 0.5 . Furthermore, changes in the correction level of $\mathrm{AO}$ and different wind velocities in different atmospheric turbulence layers at different altitudes as well as parameters describing the experimental setup of the AO have to be considered for simulation purposes.

Here, we account for some of the above problems and present two modified versions of the Göttingen speckle masking code. Their application to AO-supported observations, performed in June 2004 at the SST, is explained and the results are compared with each other and the ones obtained by the conventional method. In reconstruction $A$ - the conventional method we neglect the use of AO completely and presume only isotropic atmospheric influence. As a first approach, reconstruction $B$ allows for a radial dependence of the Fried parameter and thus of the $S T F$, but still assumes purely atmospheric influence on the transfer function. In reconstruction $C$ we account for the influence of Adaptive Optics correction, applying newly simulated $S T F$ s that reflect the partial character of the wave front (WF) correction and the according WF error statistics. As a starting point for our STF calculations, which are described in Sect. 3.3, the TurbuLenZ software package (developed at the MPIA in Heidelberg by Berkefeld \& Weiss) has been chosen. It is used to generate atmospherically distorted wave front phase screens which are then measured by the wave front sensor (WFS). The code provides the possibility to model an arbitrary number of atmospheric turbulence layers, which can be characterised independently by assigning freely selectable heights, different values of $r_{0}$, outer scales of turbulence and wind velocities to each of them. The optional declaration of an arbitrary number of viewing directions, for which the resulting wave front is to be calculated, is also supported by the code.

\section{Observations}

The active region NOAA AR0636 at $6^{\circ} \mathrm{S}$ and $20^{\circ} \mathrm{E}$ was observed on 21 June 2004 with the SST, La Palma using AO (Scharmer et al. 2003a,b). We image simultaneously through $11.56 \AA, 9.1 \AA$, and $26.0 \AA$ wide filters centred at $4305.6 \AA$ (G-band), 4507.5 $\AA$ (blue continuum), and 6020.0 $\AA$ (red continuum), respectively. A strictly simultaneous time sequence of high resolution images was taken spanning 8 min from 13:22 UT to $13: 30 \mathrm{UT}$. Bursts of 100 frames per $30 \mathrm{~s}$ with exposure times of $10.7 \mathrm{~ms}, 13 \mathrm{~ms}$, and $14.3 \mathrm{~ms}$ in the $G$-band, blue and red continuum have been taken to provide the possibility of postfacto speckle reconstruction. The images were acquired using three Kodak Megaplus 1.6 CCD detectors, with a dynamic range of 10 bits and $1536 \times 1032$ pixels. The pixel size of the CCD corresponds to 0 ' $^{\prime} 04$ (the spatial resolution of the reconstructed images is approximately $0 .{ }^{\prime} 1$ ). Only the best burst at each wavelength was used.

\section{Speckle reconstruction}

\subsection{Conventional method - reconstruction $A$}

The reconstruction of images is performed in the Fourier domain, using the image equation

$F\left(s, t_{i}\right)=F_{0}(s) \cdot O T F\left(s, t_{i}\right)$

where $s$ is the spatial frequency coordinate, $F$ the Fourier transform of the $i$ th image recorded at the time $t_{i}, F_{0}$ the Fourier transform of the observed object, and OTF the optical transfer function.

Fourier phases are recovered from $N=100$ short-exposure images using the speckle masking method in the conventional way (Weigelt 1977; see also de Boer \& Kneer 1992; and de Boer 1996) as the AO influence on image formation does not affect this part of the object information recovery.

The Fourier amplitudes of the reconstructed image, however, result from Labeyrie - and spectral ratio methods (Labeyrie 1970; von der Lühe 1984) which consider only atmospheric influences to model the statistical averages of the OTF. We have to implement AO induced changes in the modelling of the OTF.

The spectral ratio $\epsilon$ tells us which model OTF is appropriate for the reconstruction, since it is calculated from observational data as well as from the model functions:

$\epsilon(s)=\frac{\left|\left\langle F\left(s, t_{i}\right)\right\rangle\right|^{2}}{\left\langle\left|F\left(s, t_{i}\right)\right|^{2}\right\rangle}=\frac{\left|\left\langle O T F\left(s, t_{i}\right)\right\rangle\right|^{2}}{\left\langle\left|O T F\left(s, t_{i}\right)\right|^{2}\right\rangle}$,

where $\langle\ldots\rangle$ stands for the arithmetic average over all images $i$ of one speckle burst (ensemble average).

The $S T F$ is the ensemble average of the squared modulus of the short-exposure transfer functions,

$\operatorname{STF}(s)=\left\langle\left|O T F\left(s, t_{i}\right)\right|^{2}\right\rangle$,

and is unknown a priori. Yet it has to be modelled as it is used for the amplification of the average power spectrum of the image series, yielding the power spectrum of the "true image":

$\left|F_{0}(s)\right|^{2}=\frac{\left\langle\left|F\left(s, t_{i}\right)\right|^{2}\right\rangle}{S T F(s)}$

For observations not supported by AO the spectral ratio is characterised only by the normalised Fried parameter $\alpha=r_{0} / D$, where $D$ is the aperture of the telescope. Thus, the spectral ratio method determines the specific model STF that suits best the (average) atmospheric properties during observation, as in this model the $S T F$ depends only on $\alpha$ (Korff 1973).

The speckle image reconstruction method requires isoplanar conditions. Since the FOV exceeds by far the isoplanatic patch, the images have to be divided into isoplanatic subfields, which have to be reconstructed separately. For the present data set, 187 subfields of $55^{\prime \prime} 12 \times 55^{\prime \prime} 12(128 \times 128$ pixels $)$ have been chosen.

\subsection{Consideration of a field dependence of the Fried parameter - reconstruction $B$}

In the conventional method $(\operatorname{method} A)$, isotropic and purely atmospheric influences are considered, thus $\epsilon$ can be determined 
as the average over all subfields to reduce noise and uncertainties for larger wave numbers. However, in the case of AO supported observations, a radial variation of $\epsilon$ is observed. We have field varying wavefront distortions, caused by the high altitude turbulence layer. An optimal correction is possible only for the AO lockpoint region, because only for this direction the distortion measurements of the WFS are valid. Thus, with increasing distance from the lockpoint the AO compensation of high altitude turbulence is less and less efficient. While the Fourier phases are not affected by this variation, the Fourier amplitudes of the object do change, because the true intensity of the observed object is estimated by the power spectrum of the speckle data and after division by the model $S T F$ s. The variation of $\epsilon$ results in a wrong estimation of the true $r_{0}$ in method $A$ and hence of the resulting $S T F$ for certain parts of the image. Therefore, the use of field dependent $S T F$ s is mandatory for AO supported observations. As a first attempt we assume, in method $B$, that the field dependent WF correction by AO changes only the estimated value of the Fried parameter for different positions over the FOV, thus the estimation of $r_{0}$ with the spectral ratio method is done for different viewing directions. We address this problem in the following way.

Firstly, to identify the position of the AO lockpoint, the residual image motion of the individual isoplanar subfields over $N=100$ time steps is measured after the compensation of the overall image motion. The lockpoint of the AO is assigned to the subfield with the lowest variance of the residual image jitter. In Fig. 1 the measured normalised variance values are presented for the different subfields. At the SST both the tip-tilt (TT) mirror and the DM have an independently selectable lockpoint. For the presented data it was taken care during the observation to have both lockpoints coincide as much as possible.

Secondly, to account for the radial dependence, the following geometry is assumed: a circle with a diameter of 5.' 12 (hereafter referred to as annulus 1) is centred on the lockpoint of the AO. This selected circular area corresponds fairly well to the central pore, which during observation was the actual target of the wavefront sensor. It is surrounded by 6 concentric annuli, each of width 5 .' 12, an estimate of the isoplanatic angle. The annuli are considered to be areas, where no change of the spectral ratio is assumed, see Fig. 2 (right panel).

Thirdly, the centre positions of the isoplanar subfields (presented as bright points superimposed on the images of Fig. 2) are calculated. The spectral ratios of only those subfields that have their centre position inside single annuli, are averaged for the determination of $r_{0}$. The number of subfields assigned to the individual annuli can be seen in Table 1 .

Figure 3 depicts the change of the spectral ratio with increasing radial distance from the AO lockpoint. The azimuthally averaged spectral ratios vs. relative wave number are presented. Note that the spectral ratio of annulus 1 (lockpoint area) results from one single subfield and has been smoothed by a boxcar of 7 pixels to reduce noise. For all other annuli the averaging of $\epsilon$ suppresses the noise level sufficiently for a safe estimate of $r_{0}$, which follows from the comparison of the measured and the model spectral ratios. Those spatial frequency values $k_{\epsilon}$ are compared where $\epsilon$ drops to a value of $0.5,0.3,0.2$ or 0.1 . For these estimates of the seeing cut-off frequency we have tabulated values for the coefficients $a$ and $b$ in the relation with the Fried parameter, which is a power law $r_{0}=a \cdot k_{\epsilon}^{\mathrm{b}}$ that depends slightly on the chosen values of $\epsilon$. Thus, one can select either the maximum, minimum or mean value of the different estimates for $r_{0}$ and the appropriate model STF for the subsequent reconstruction. We choose the maximum value to avoid an over-amplification of

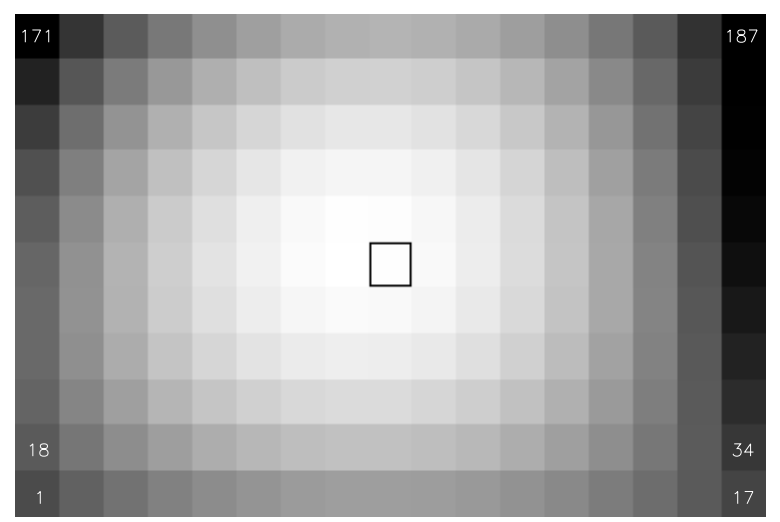

Fig. 1. Normalised variance of image position over 100 time steps for the 187 individual subfields. Darker shades correspond to higher variance. The small black box indicates the subfield with the smallest image motion, containing the AO lockpoint. The variance values are calculated from overlapping subfields of $5^{\prime \prime} 12 \times 5^{\prime \prime} .12$ size. The overlap is not considered in this graphical representation.

the Fourier amplitudes, i.e. the reconstructed intensities for the real object. Another possible method is used by Denker et al. (2004). They look at the decrease of the spectral ratio and use the crossing of a linear fit with the frequency axis as their estimate of the seeing cut-off frequency. This results in a higher estimate for the Fried parameter and therefore in a more conservative intensity amplification than in our method. However, using Adaptive Optics the changed shape of $\epsilon$ can lead to misinterpretations in both methods. For example, at the lockpoint region (see Fig. 3), the decrease of the spectral ratio is steepened and located at higher wave numbers than in the uncorrected case. There, both estimates of $r_{0}$ may result in too large values, thus yielding a wrong selection of the $S T F$ used for reconstruction.

In Table 2 the determined Fried parameters at different wavelengths are presented for annuli 1 to 7 , together with the ones obtained from method $A$. A rapid decay of $r_{0}$ with increasing radial distance from the lockpoint is observed at all wavelengths. Yet further out, from the 5th annulus on we see a stagnation of high altitude turbulence compensation. The values of $r_{0}$ remain surprisingly high and tend to become constant. This can be explained considering the low altitude turbulence compensation: the (stronger) turbulence layer near the telescope entrance window affects the wave fronts of all viewing directions equally and to a large amount, including the WF seen by the WFS. Thus, a good compensation of wave front deformations stemming from this layer is achieved over the whole FOV. This is the reason why a significant improvement also at the outer parts of the image is observed. In Fig. 4 (left panel) the corresponding STFs used for reconstruction $B$ are shown together with the one used in method $A$ for the case of the $G$-band data.

\subsection{Accounting for complete AO influence on the STF - reconstruction $C$}

The instantaneous $O T F$ s, which are calculated via autocorrelation of the wave fronts at the telescope pupil, are affected in a different way for each spatial frequency, depending on the number of corrected Zernike modes. Higher Zernike modes are more sensitive to different viewing angles because the corresponding turbulence patterns have smaller scales and decorrelate faster, i.e. at high spatial frequencies the correction decays faster with radial distance from the lockpoint than at low spatial 


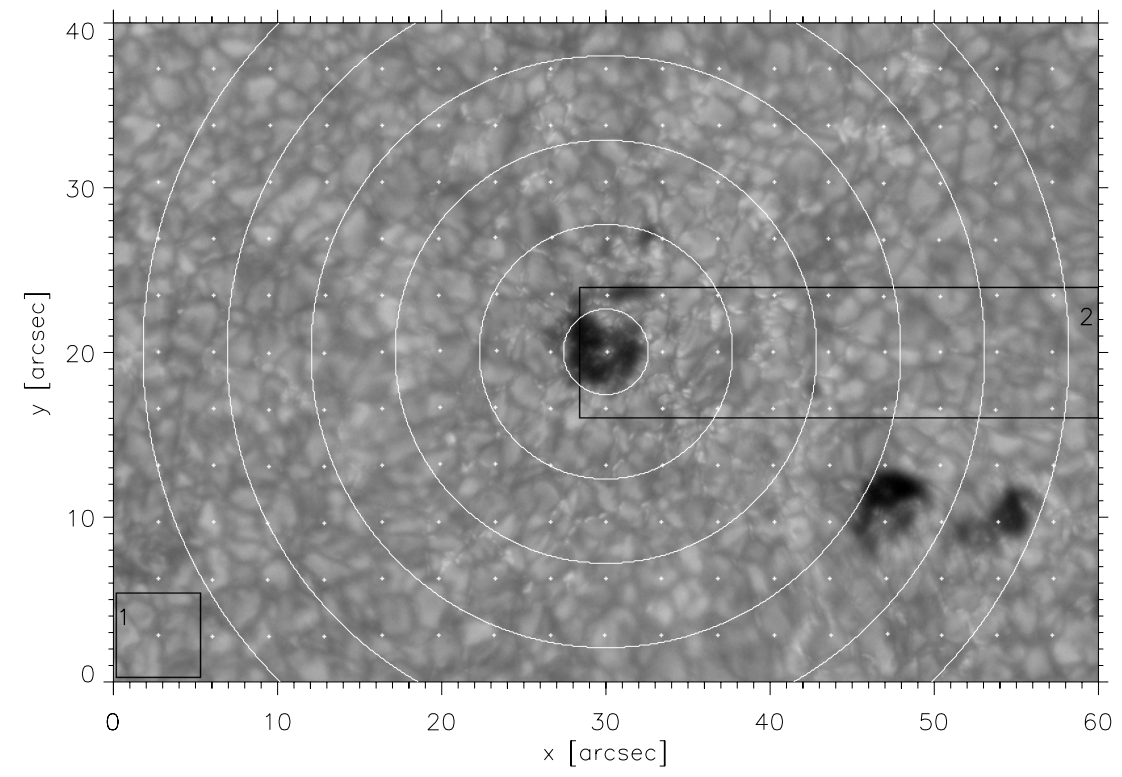

Fig. 2. $G$-band raw image of the active region NOAA AR0636 at $6^{\circ} \mathrm{S}$ and $20^{\circ}$ E observed on 21 June 2004 with the Swedish 1-m Solar Telescope (SST), La Palma. The total FOV is $60^{\prime \prime} \times 40^{\prime \prime}$. White contour circles depict the geometrical constraint used in methods $B$ and $C$ for a better modelling of the field dependence of AO correction. The centre circle (annulus 1) with 5'.12 diameter overlaps with the lockpoint area of the AO, whereas surrounding concentric annuli of $5^{\prime \prime} .12$ width are considered to be areas where no change of spectral ratio is assumed. The white points superimposed on the images denote the centre positions of the individual isoplanar subfields of $128 \times 128$ pixels $\left(5^{\prime \prime}\right.$. $12 \times 5{ }^{\prime \prime}$. 12$)$ to be reconstructed. The black box 1 indicates one of these isoplanar subfields. Box 2 borders the subfield of the total image presented in Fig. 5. See text for a detailed description.

Table 1. Number of subfields used for the calculation of the average $\epsilon$ of the individual annuli.

\begin{tabular}{cccccccc}
\hline \hline Annulus: & 1 & 2 & 3 & 4 & 5 & 6 & 7 \\
\hline Number of subfields: & 1 & 20 & 24 & 44 & 41 & 41 & 16 \\
\hline
\end{tabular}

Table 2. Fried parameters at different wavelengths, calculated with the spectral ratio method. Maximum values of $r_{0}$ of the individual annuli for method $B$ and the $r_{0}$ for method $A$, obtained by averaging $\epsilon$ over the whole FOV. Wavelengths $\lambda$ in $\AA, r_{0}$ in $\mathrm{cm}$.

\begin{tabular}{cccccccc|c}
\hline \hline Annulus: & 1 & 2 & 3 & 4 & 5 & 6 & 7 & $A$ \\
\hline$r_{0}$ at $\lambda$ 4305: & 48 & 34 & 30 & 27 & 26 & 25 & 23 & 27 \\
$r_{0}$ at $\lambda$ 4507: & 52 & 39 & 35 & 32 & 30 & 30 & 30 & 32 \\
$r_{0}$ at $\lambda$ 6020: & 64 & 54 & 49 & 45 & 43 & 43 & 41 & 45 \\
\hline
\end{tabular}

frequencies. Changes in the statistical properties of the wave front errors by AO compensation translate into a shape of the $S T F$ that cannot be reproduced by a variation of the Fried parameter alone. Still, for lower modes, the correction is significant up to higher radial distances. In the case of tip-tilt corrected shortexposure images, an $\mathrm{AO}$ correction of even the three remaining lowest Zernike modes (defocus and astigmatism) reduces the variance of the residual WF deformation by more than $50 \%$ (Noll 1976). For method $C$ we therefore simulated the AO compensation of atmospherically distorted wave fronts, regarding the seven viewing directions already introduced with model $B$, and calculated the corresponding $S T F \mathrm{~s}$. We take reconstruction $C$ as our best estimate of the real situation.

Wave front phase screens at the telescope pupil are calculated with the TurbuLenZ simulation code. The main settings applied can be summarised as follows.

- A two-layer model for atmospheric turbulence $(0 \mathrm{~m}, 6000 \mathrm{~m})$ is considered, thus for both layers a certain turbulence

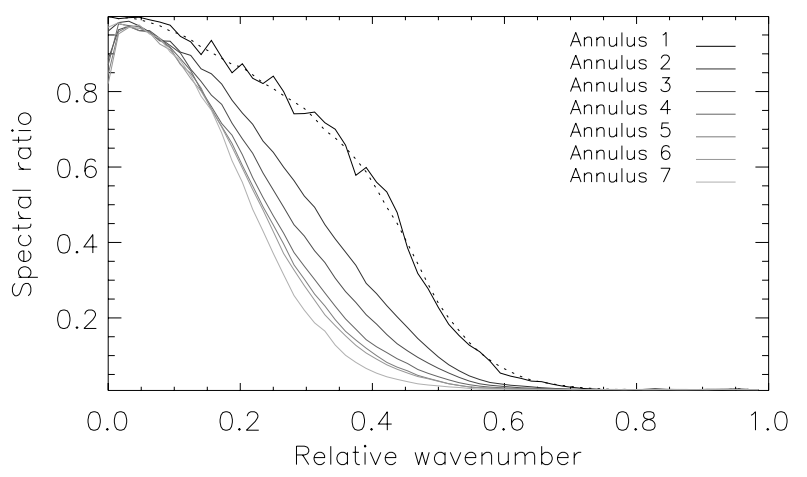

Fig. 3. Azimuthally averaged spectral ratios $(\operatorname{method} B)$ of the 7 individual annuli vs. relative wave number. The spectral ratio of annulus 1 (lockpoint region) results from one single subfield and has been smoothed by a boxcar of 7 pixels in wave number to reduce noise (dashed line).

strength must be defined. The effective $r_{0, \text { eff }}$ of the entire atmosphere can be computed from $r_{0 \text {, low }}$ and $r_{0 \text {,high ("Fried pa- }}$ rameters" characterising the low and high layer separately) according to

$r_{0, \text { eff }}=\left[r_{0, \text { low }}{ }^{-5 / 3}+r_{0, \text { high }^{-5 / 3}}\right]^{-3 / 5}$.

- For the $G$-band $r_{0, \text { high }}=50 \mathrm{~cm}$ has been assumed, accounting for the excellent seeing conditions during the observational run. This value seems appropriate for AO-corrected data that show a moderate dependence on the viewing angle and the amount of increasing differential image motions with growing radial distance from the AO lockpoint in the FOV (T. Berkefeld, private communication). According to the scaling law

$r_{0, \mathrm{eff}} \propto \lambda^{6 / 5}$, 

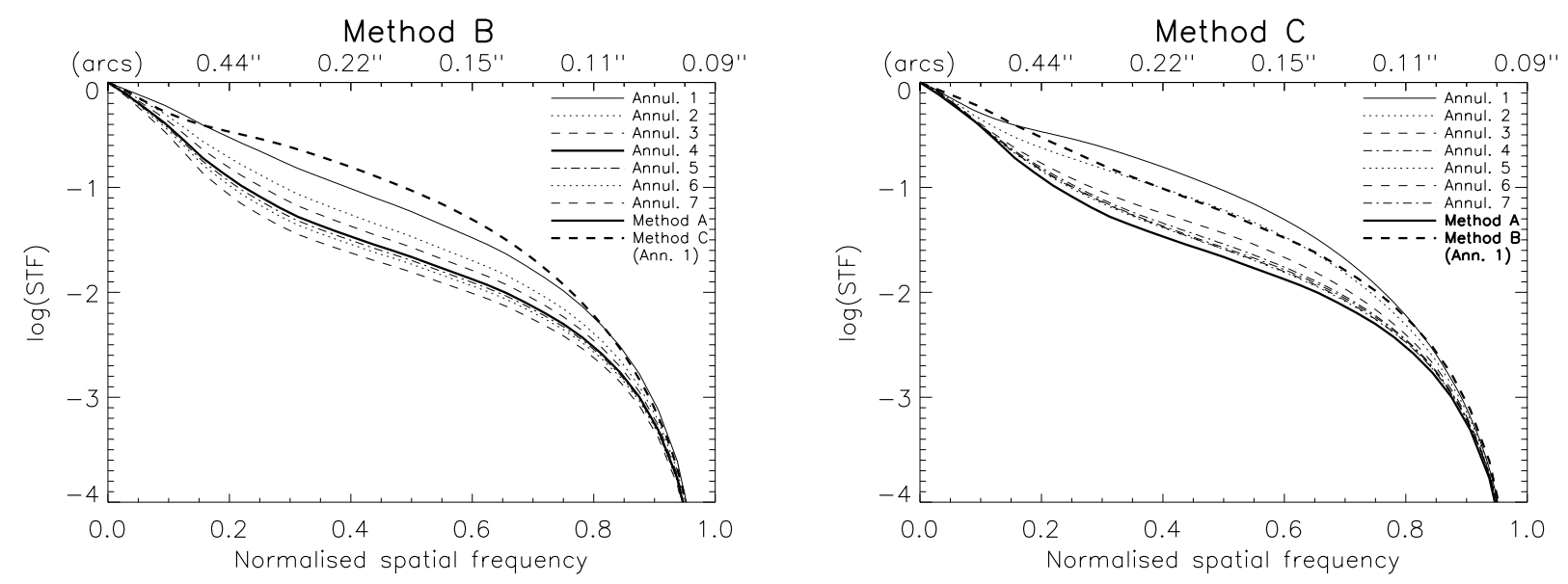

Fig. 4. Logarithmic representation of the model $S T F \mathrm{~s}$ used, with spatial frequency normalised to the diffraction limit $D / \lambda$ of the telescope. Left panel: model $S T F$ s used for reconstruction $B$ of the $G$-band image. Here, only a change of the Fried parameter with radial distance from the AO lockpoint is considered. Right panel: $S T F$ s used for reconstruction $C$. Influences of various AO parameters on the $S T F$ s are taken into account. For reference, the $S T F$ used for reconstruction $A$ is plotted in both panels (thick solid line). Additionally, the lockpoint-STFs of method $B$ and $C$ are represented with thick dashed lines in the right and left panel, respectively.

Fried parameters $r_{0, \text { high }}=53 \mathrm{~cm}$ and $r_{0, \text { high }}=75 \mathrm{~cm}$ follow for the blue and the red continuum, respectively. We adopt the Fried parameters measured at the outermost annulus in the FOV as $r_{0, \text { eff }}$ (see Table 2). The AO correction there is assumed to be negligible. Thus after Eq. (5), $28 \mathrm{~cm}, 40 \mathrm{~cm}$ and $54 \mathrm{~cm}$ for $r_{0, \text { low }}$ are estimated for the different wavelengths. This assumption is necessary, although not completely fulfilled. As stated above in Sect. 3.2, even at the outermost parts of the image a certain correction of the ground layer turbulence is provided by the AO. This results in an overestimation of $r_{0, \mathrm{eff}}$. However, towards the outer image parts, less and less information about the high altitude turbulence is available from the wave front sensor. The actual WF deformations that affect these image regions become more and more statistically independent from the deformation sensed. Thus, the calculated shape of the DM induces further wave front errors there, leading to an underestimation of the atmospheric seeing condition. This compensates partly for the former overestimation of the low layer turbulence strength $r_{0, \text { low }}$. Similarly, a consequence of our lack of knowledge about the (exact) AO activity during each burst is that we probably deal with a mixture of variably corrected images. For the estimation of the true seeing condition this is advantageous, as the true atmospheric statistics are visible, at least for some images in a burst, and so lessens the overestimation of $r_{0}$ as well. To avoid the present problems of Fried parameter estimation, on the one hand, simultaneous observations without $\mathrm{AO}$ correction could be used to obtain the true $r_{0 \text {,eff }}$, averaged over the time of one burst. On the other hand, with information about the number of corrected Zernike modes during observation, which was not available in our case, we could calculate for each image the theoretically expected value of the variance of the corrected wave fronts. The time dependent, effective Fried parameter could then be derived, as $r_{0, \text { eff }}$ can also be defined as the diameter of a circular part of a wave front, with variance equal to $1 \mathrm{rad}$. A corresponding mixed series of WFs could then be simulated and corrected by our AO simulation code and result in a more specific estimate of the representative $S T F$. However, this to make sense, the estimation or measurement of the basic atmospheric model parameters have to be robust and accurate. Yet we expect that only minor differences would be noticed, as only bursts with stable AO correction are made use of. Speckle bursts containing several uncompensated frames are usually discarded.

- Mean wind velocities and directions were taken from meteorological data for the day and time of the observations. However, this can only provide a very rough and average approximation to the situation at the time and location of data collection, since these conditions are highly variable in space and time. Also the velocities are very different for the altitudes of our assumed two turbulence layers. We conclude that it is safer to assume no wind at all.

- Outer scales of turbulence are assumed to be $6 \mathrm{~m}$ and $50 \mathrm{~m}$ for the lower and the higher layer, respectively.

- For seven different viewing directions, corresponding to the seven annuli already used in method $B$, the according part of the phase screens of the high turbulence layer is propagated down to the telescope (Fresnel propagation), to account for anisoplanatic effects, and added to the phase retardation induced by the ground layer.

Secondly, models for the WF measurement and for the WF correction are both implemented in our simulation package. With respect to the actual optical setup of the AO system used at the SST, the pupil diameter of $1 \mathrm{~m}$, the hexagonal geometry of the 36 lenslet Shack-Hartmann WFS and the configuration of the 35 actuators of the deformable mirror (DM) are incorporated. In the simulation as in the experimental setup, the WFS measurement and the actuator signals for the DM have to be calibrated with respect to each other. This procedure yields a control matrix that translates the measured aberrations into the mirror deformation necessary to correct the wave front as well as possible. The measurements of the average tilt of the WF over each subaperture is modelled directly by fitting planes (tilted in $x$ - and $y$-direction) to the WF parts and calculating the corresponding image shift in the focal plane of the WFS. The residual wave front is then given by the difference between the original wave front and the resulting shape of the DM.

Thirdly, we turn towards the calculation of the STF. 100 independent wave front realisations were generated and the simulated AO correction performed on each of them, to simulate the individual short-exposures of our speckle bursts and to have the same statistical averages of wave front errors. 


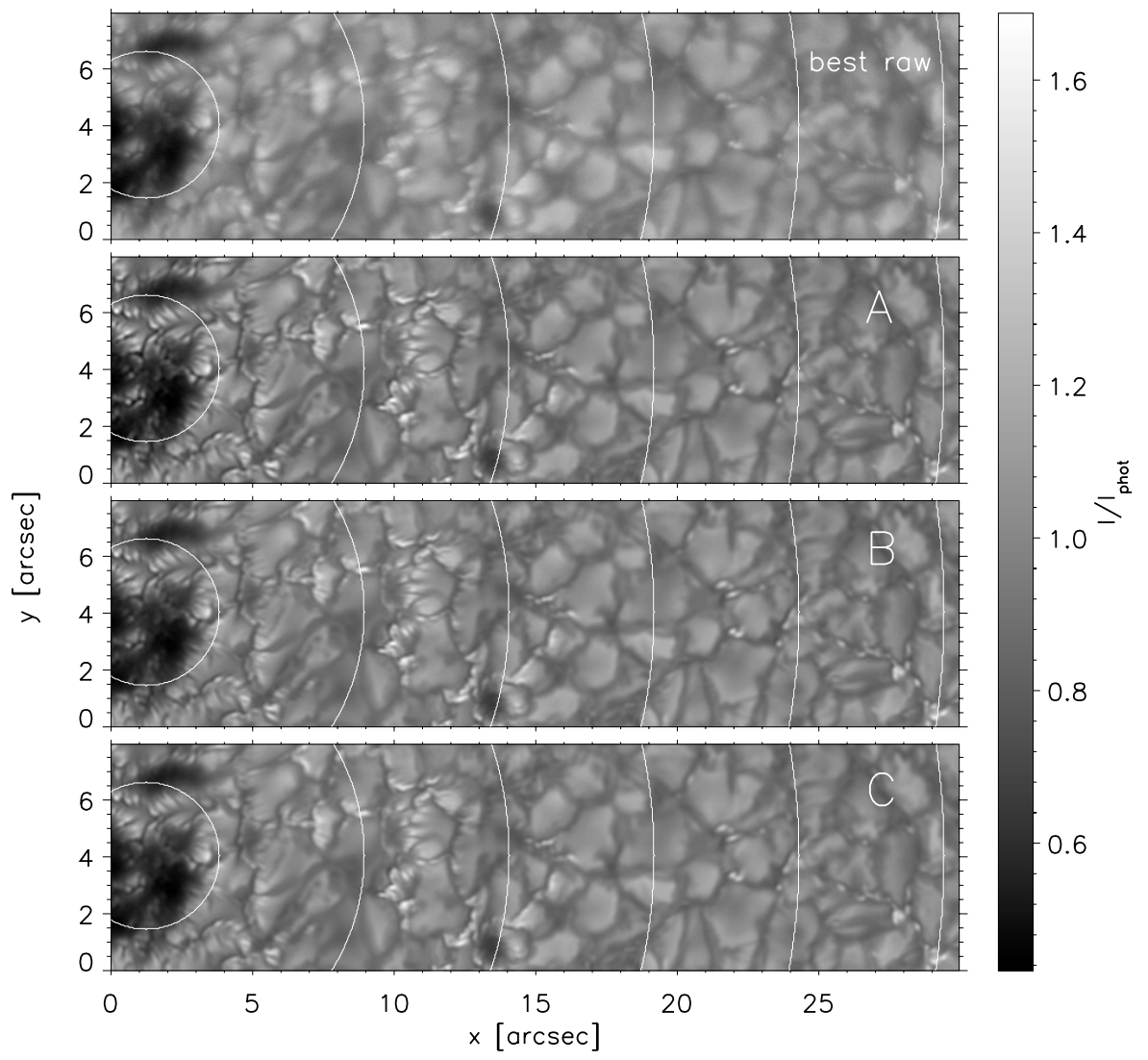

Fig. 5. From top to bottom: part of the best raw image (see Fig. 2, Box 2 ) and corresponding parts of the speckle reconstructed images of the active region NOAA AR0636 after applying reconstruction $A$, reconstruction $B$ and reconstruction $C$, respectively. All images are normalised to the mean intensity of the quiet photosphere. The pore to the left represents the lockpoint (centre of the total FOV), the right edge of the panels coincide with the right margin of the large image. Subfield size $30^{\prime \prime} \times 8^{\prime \prime}$. Individual annuli are represented by white contours.

The corresponding instantaneous $O T F$ s are calculated via autocorrelation of the residual wave fronts. The STF follows as the average of the squared absolute $O T F$ s (see Eq. (3)).

The simulations are done for all three wavelengths under study and calculated for each of the seven annuli, as described above, thus for these areas we obtain individual speckle transfer functions, presented for the case of the $G$-band in Fig. 4 (right panel). The difference between the $S T F$ s used for the several reconstruction methods will be discussed in detail in the following section.

\section{Results and discussion}

Figure 5 shows a part (see Fig. 2, box 2) of the resulting speckle reconstructed images of the active region NOAA AR0636, after applying different reconstruction methods. The three methods provide similar results, since the Fourier phase reconstruction is not altered by $\mathrm{AO}$ usage and therefore the positions and shapes of all features are equal in all images. A closer inspection, however, reveals some difference in contrast and absolute brightness at different spatial scales, depending on the region in the FOV. Therefore we show the differences of every pair of reconstructions for better visualisation in Fig. 6 .

The upper panel of Fig. 6, showing the difference between reconstruction $B$ and $A$, depicts the effects introduced when a simple radial variation of the Fried parameter is considered. In both the centre part and the outer region of the FOV, differences are visible. Only at annulus 4 , where the Fried parameter of method $B$ is very similar to the average value of method $A$, we find almost identical intensity values. Throughout the central region we note an overestimated contrast in reconstruction $A$, especially pronounced for the smallest structures (modulus of intensity differences up to $16 \%$ ). This effect is strongest at the lockpoint and diminishes at a distance of about $10^{\prime \prime}$ to the lockpoint. At the outer parts of the image we observe a somewhat higher contrast in reconstruction $B$ with respect to $A$ (modulus of intensity differences up to $5 \%$ ) at all spatial frequencies (see therefore also Fig. 7, upper panel).

One can understand this behaviour by looking at the respective transfer functions (Fig. 4, left panel): in general, a larger estimate of $r_{0}$ leads to a higher $S T F$, i.e. with higher signal at all frequencies. For intensity amplification we divide the image spectrum by the $S T F$, thus the larger $r_{0}$ the smaller the resulting intensities. The extent of impact depends on the magnitude of $r_{0}$ and on the difference to the average Fried parameter of method $A$. Therefore, the underestimation of $r_{0}$ in the central region around the lockpoint in method $A$ leads to an overestimation of contrast. For the outer parts of the image, $r_{0}$ is slightly underestimated by method $B$ thus yielding somewhat too large a contrast. However, the contrast is not only influenced by an overall enhancement of intensities, which would correspond to a simple shift of the $S T F$ along the $y$-axis, but also by the relative differences of amplification for different frequencies.

We discuss the differences between reconstruction $C$ and $A$ (middle panel of Fig. 6). Around the lockpoint region, we have a result similar to the difference $B-A$, although the modulus of the intensity differences visible in $C-A$ is larger (up to $22 \%$ ) and significant up to a distance of about $15^{\prime \prime}$, especially for the 


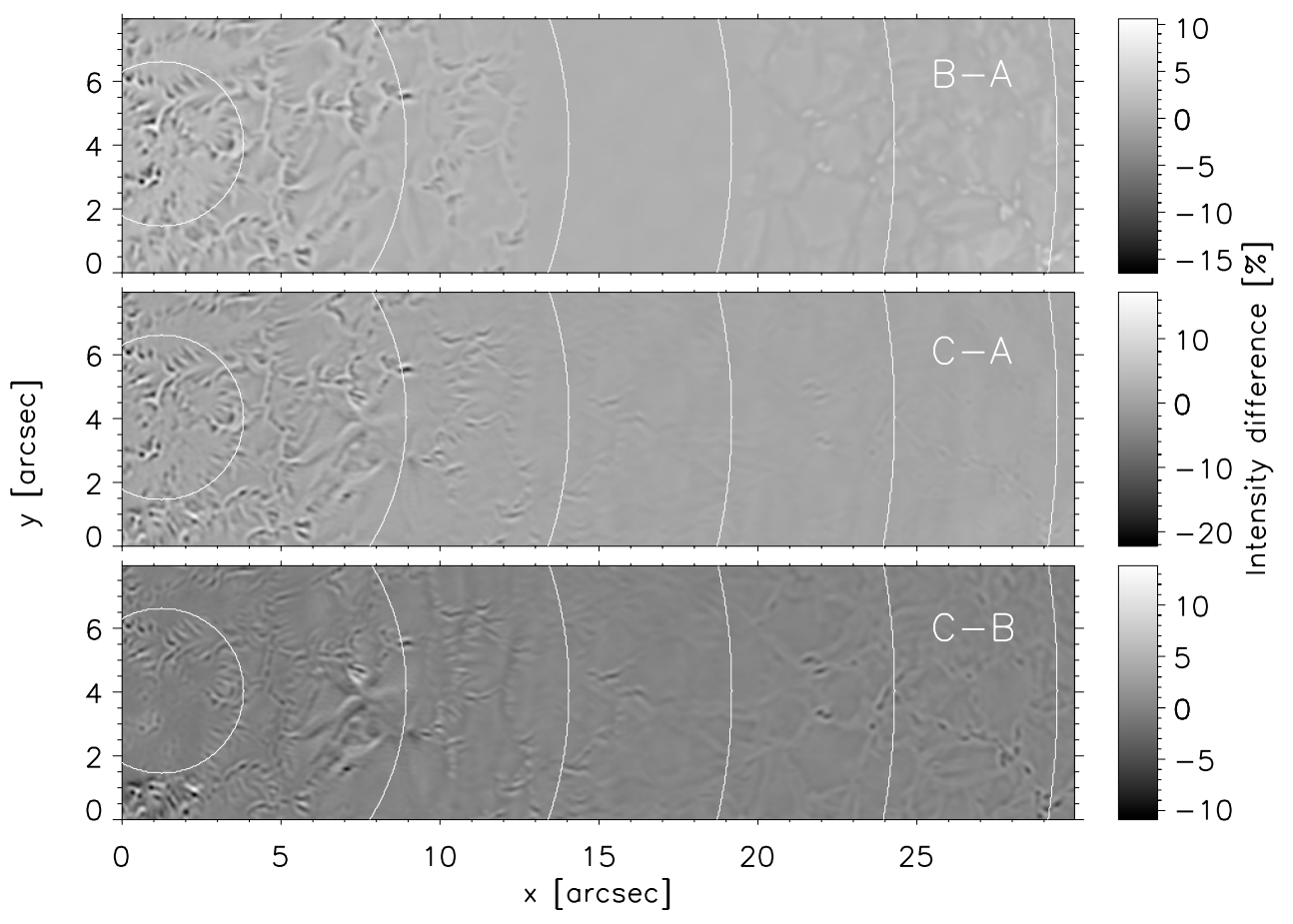

Fig. 6. Differences between the three speckle reconstructed images presented in Fig. 5 as percentages relative to the mean intensity of the quiet photosphere $\left(I_{\text {phot }}\right)$. $B-A$ (upper panel), $C-A$ (middle panel), $C-B$ (lower panel). Individual annuli are represented by white contours.

smallest structures. Consequently, reconstruction $C$ suppresses the overestimation of intensities around the lockpoint region even more than method $B$. At the outer annuli, reconstruction $C$ reveals a similar amplitude magnification as observed in method $A$, unlike the one visible in the difference $B-A$. The observed intensity differences occur mainly at the smallest scales and rarely exceed $\pm 3 \%$ and thus lie inside the confidence interval of speckle reconstruction for intensities (see also Fig. 7, middle panel).

For the STFs at the lockpoint (Fig. 4, right panel), the same argumentation as for the difference $B-A$ holds. Furthermore, as all $S T F$ s used in reconstruction $C$ lie above the corresponding ones of method $B$ at smaller scales, a less strong intensity amplification of these structures throughout the FOV is observed.

The lowest panel in Fig. 6 displays the differences between the two new methods. At the lockpoint region intensity differences of up to $13 \%$ are restricted to two small areas in the second annulus. In the remaining area, including the outer parts of the image, the modulus of the intensity differences does not exceed $6 \%$. Reconstruction $C$ further damps the amplification of smaller structures around the lockpoint region and throughout the FOV compared to method $B$, reflecting the difference in shape of the modelled AO STFs, that is, a frequency dependent alteration of the conventional atmospheric $S T F$ s. The slightly higher contrast at all spatial frequencies at the outer annuli resulting from reconstruction $B$ is confirmed (see also Fig. 7, lower panel).

To obtain an additional, independent measure of image quality for each reconstruction we focus now on the rms contrast of the resulting images and its field dependence. In Fig. 8 we present the resulting graphs for each reconstruction. The rms values of the contrast for each annulus are calculated from the image points lying inside the individual rings. However, this calculation is not straightforward. For an active region with very dark and very bright features, the calculated rms value is very high, regardless of the image reconstruction method. Since the whole FOV of our observations is covered non-uniformly with

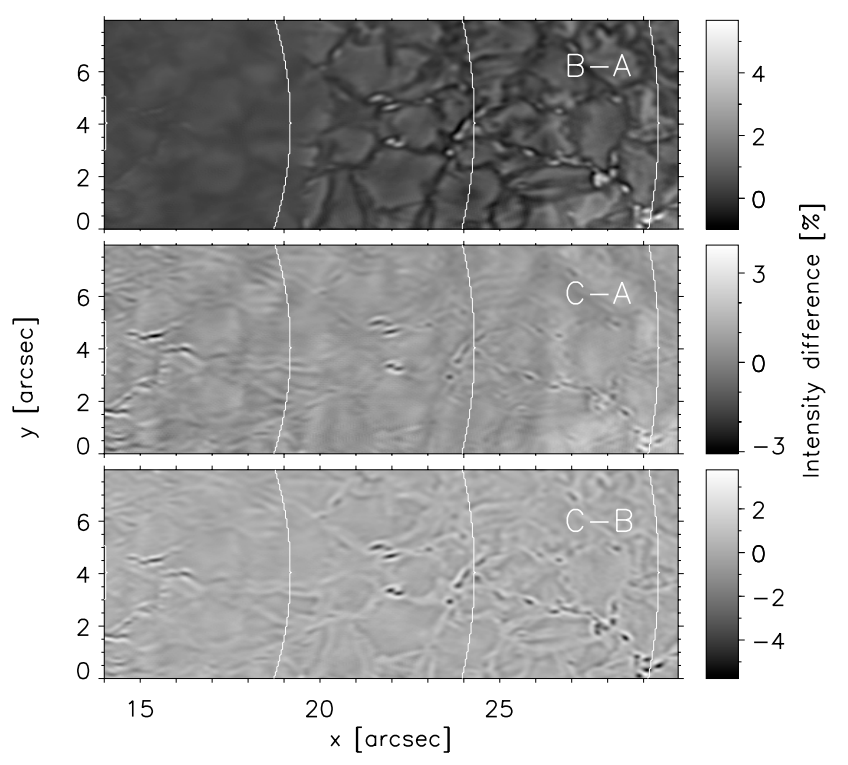

Fig. 7. Outer parts of difference images presented in Fig. 6 for a better presentation of the intensity scale as percentages relative to the mean intensity of the quiet photosphere $\left(I_{\text {phot }}\right) . B-A$ (upper panel), $C-A$ (middle panel), $C-B$ (lower panel). Individual annuli are represented by white contours.

such structures, the calculated contrast would rather reflect the image content, but not the quality of the reconstruction method. Such a measure would be more useful for data with a more homogeneous contrast of the science object, i.e. observations of a quiet solar region. Nevertheless, we tried to simulate such a quiet region by the following two constraints: firstly, we define for the whole FOV a lower threshold of $75 \%$ of the mean intensity of the quiet photosphere, to exclude the pores visible at the lockpoint and the lower right corner of the images. 


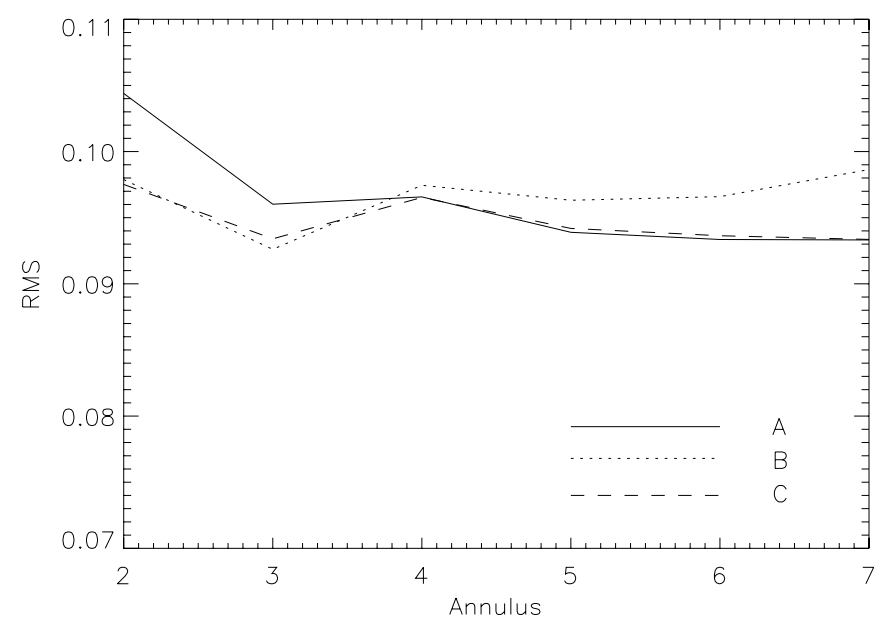

Fig. 8. Field dependence of the rms contrast for the three Speckle reconstructions $A, B$ and $C$. For the calculation we normalised the images to the mean intensity of the quiet photosphere. Since the contrast of active regions reflects predominantly the image content, we additionally left out the regions covered by pores and by structures associated with strong magnetic elements, mostly intergranular bright points and filamentary structures, to simulate a quiet photosphere. Therefore, the value for annulus 1, containing the pore used as lockpoint, is not meaningful and has been omitted.

Secondly, we tried to eliminate tracers of magnetic activity, like intergranular bright points, in each annulus separately. This was done by successively eliminating the brightest pixels together with a small neighbourhood ( \pm 3 pixels), until the procedure started to find only bright granules. Only the image region of an annulus that was left unaffected by both criteria was used for the calculation of the rms contrast. We therefore have no meaningful value for the lockpoint, where the pore and bright signatures of magnetic fields lead to the exclusion of almost all image points. Nevertheless, the resulting field distribution of contrast, shown in Fig. 8 for the three different reconstructions, confirms our previous discussion of the different reconstruction methods and verifies our new method $C$, as it leads to the most uniform image quality, in terms of rms contrast, throughout the FOV. However, as a first step, method $B$ correctly lowers the contrast in the lockpoint region, although it leads to a slight overestimation at the outer parts of the FOV.

\section{Conclusions}

Two modified methods ( $B$ and $C$ ) for the speckle reconstruction of AO supported observations have been developed and applied for a more realistic estimation of the photometric intensities in reconstructed images. We have compared their results with each other and with that obtained by the conventional method $A$ (de Boer 1996) in terms of field dependent intensity differences and rms contrast. In method $A$ one $S T F$ is used for reconstruction, estimated from a Fried parameter averaged over the whole FOV under the assumption of pure atmospheric influence. Our first approach, method $B$, allows for a spatial variation of the $S T F$ in the FOV as a function of radial separation from the lockpoint of the AO. This is done by estimating individual Fried parameters $r_{0 \text {,eff }}$ for annular image parts around the lockpoint. However, method $B$ still assumes pure atmospheric statistics for the wave front distortions and the subsequent calculation of the STF. Our second approach, method $C$, employs simulated model STFs considering the influence of Adaptive Optics WF correction on the shape of the transfer functions. We also addressed the dependence on the viewing angle, since the corresponding parts of the WF, that affect image regions away from the lockpoint, and the measured part of the WF, seen by the WFS in the lockpoint direction, are increasingly decorrelated as the distance to the lockpoint increases in the FOV. This fact is seen mainly as differential image motion and a loss of image sharpness for increasing radial distance from the lockpoint in the FOV. We calculated the corresponding $S T F$ s for seven different viewing angles, each separated by 5 .' 12 .

We expect a good reconstruction to show uniform image quality. The result of method $C$ provides the most constant rms contrast throughout the FOV. As described in the previous section, we find that the conventional reconstruction method $A$ overestimates intensities in the lockpoint region and thus the rms contrast. However, in the outer parts of the image, method $A$ seems to estimate the actual $S T F$ fairly well, since the influence of $\mathrm{AO}$ is small because of decorrelation of WF and corrective shape of the DM. Compared to method $C$, method $B$ shows minor differences in the rms contrast around the lockpoint, but the rms contrast of the reconstructed images tends to rise towards the outer parts of the field of view. Additionally, frequency dependent differences as a function of the position in the FOV are observed. We conclude that the decrease of wave front compensation power across the FOV is not modelled appropriately by method $B$ and that a more accurate modelling implemented in method $C$ is required for a realistic result.

The estimated extra processing time for the generation of appropriate phase screens and the STF depends strongly on size of the simulated phase screens, i.e. the FOV and the assumed height of the upper turbulence layer. In our case the calculations for one set of STFs take about one hour on a $2.0 \mathrm{GHz}$ CPU, which is comparable to the time needed for the standard speckle algorithm. To pre-calculate a library of phase screens only makes sense if the selection of few measurable parameters is sufficiently correct. This would reduce the calculation time to about twenty minutes. For more individual conditions, like wind velocities, a direct calculation is, of course, preferable because of the large number of parameter combinations and the amount of disc space and calculation time needed.

The modelling of anisoplanatic STF depends on the height and strength of the upper turbulence layer. These values cannot be retrieved separately from the observed differential image motion. An accurate determination of the height would be possible if we have available either a second WFS (Multi-Conjugated Adaptive Optics, MCAO) or a larger anisoplanar FOV in a single WFS and thus the corresponding Fried parameter of this layer could be measured/calculated.

For the estimation of the overall (effective) Fried parameter we envision to gather extra data from the AO activity during the observational runs, i.e., the WFS data together with the control signals sent to the DM. This would allow an estimation of the residual WF errors and thus the estimation of the overall atmospheric conditions. Procedures for the reduction of this kind of data are presently being developed.

Additionally, for the spectral ratio method (von der Lühe 1984) the atmospheric turbulence strength and the level of AO correction interfere in effect and cannot be discerned unambiguously. The AO correction level and the number of corrected Zernike modes should be recorded as well, to allow a more precise simulation of the STFs. This information was not available in our case. The presented data were, however, collected under very good seeing conditions and the best speckle burst 
was selected. Therefore, we assumed a constant and optimal WF compensation.

The image quality and resolution enhancement should be quantified more accurately in future studies that allow comparisons with the uncorrected case and that should be repeated on more homogeneous science targets than in the presented data. This is of importance since the effects of image improvement are manifold and depend on the spatial frequency. This complicates the characterisation of the AO influences but points out the importance of our new $S T F$ s, derived from simulation, for an accurate consideration of AO-induced changes for the speckle image reconstruction algorithm.

Acknowledgements. We thank the referee, Dr. M. Löfdahl, for careful reading and for constructive comments which helped to improve the quality of this publication. We thank Prof. O. von der Lühe for his general guidance during the development of the AO simulation code, Prof. F. Kneer and Dr. T. Berkefeld for critical and helpful comments, F. Wöger for clarifying discussions of technical details. K.G.P. thanks the Deutsche Forschungsgemeinschaft for support through grant KN 152/29-1. MS gratefully acknowledges a $21 / 2$ years fellowship of the Graduiertenkolleg GRK 140 Strömungsinstabilitäten und Turbulenz by the Deutsche Forschungsgemeinschaft. The data were obtained during an observing campaign, together with M. Sobotka, financed by the European Union (OPTICON Trans-National Access Program). The Swedish Solar Tower is operated by the Institute for Solar Physics of the Royal Swedish Academy of Sciences at the Observatorio Roque de los Muchachos on La Palma of the Instituto de Astrofísica de Canarias.

\section{References}

Al, N., Bendlin, C., Hirzberger, J., Kneer, F., \& Trujillo Bueno, J. 2004, A\&A, 418,1131

Bello González, N., Okunev, O. V., Domínguez Cerdeña, I., Kneer, F., \& Puschmann, K. G. 2005, A\&A, 434, 317 de Boer, C. R. 1996, A\&AS, 120, 195

de Boer, C. R., \& Kneer, F. 1992, A\&A, 264, L24

Denker, C., Mascarinas, D., Xu, Y., et al. 2004, Sol. Phys., 227, 2

Domínguez Cerdeña, I., Sánchez Almeida, J., \& Kneer, F. 2003a, A\&A, 407, 741

Domínguez Cerdeña, I., Kneer, F., \& Sánchez Almeida, J. 2003b, ApJ, 582, L55

Fried, D. L. 1966, J. Opt. Soc. Am., 56, 1372

Fusco, T., Conan, J. M., Michau, V., Mugnier, L. M., \& Rousset, G. 1999, Opt. Lett., 24, 1472

Hirzberger, J., Stangl, S., Gersin, K., et al. 2005, A\&A, 442, 1079

Korff, D. 1973, J. Opt. Soc. Am., 63, 971

Labeyrie, A. 1970, A\&A, 6, 85

Löfdahl, M. G., \& Scharmer, G. B. 1994, A\&AS, 107, 243

Noll, R. 1976, J. Opt. Soc. Am., 66, 207

Okunev, O. V., \& Kneer, F. 2004, A\&A, 425, 321

Paxman, R. G., Seldin, J. H., Löfdahl, M. G., \& Scharmer, G. B. 1996, ApJ, 466, 1087

Puschmann, K. G., \& Wiehr, E. 2006, A\&A, 445, 337

Rimmele, T. R. 2000, Proc. SPIE, 4007, 218

Rimmele, T. R., \& Radick, R. R. 1998, Proc. SPIE, 3353, 72

Rimmele, T. R., Richards, K., Hegwer, S. L., et al. 2003, Proc. SPIE, 4839, 635

Scharmer, G. B., Bjelksjö, K., Korhonen, T. K., Lindberg, B., \& Petterson, B. 2003a, Proc. SPIE, 4853, 341

Scharmer, G. B., Dettori, P. M., Löfdahl, M. G., \& Shand, M. 2003b, Proc. SPIE, 4853,370

van Noort, M., Rouppe van der Voort, L., \& Löfdahl, M. G. 2005, Sol. Phys., 228, 191

von der Lühe, O. 1984, J. Opt. Soc. Am. A, 1, 510

von der Lühe, O., Soltau, D., Berkefeld, T., \& Schelenz, T. 2003, Proc. SPIE, 4853, 187

Voitsekhovich, V. V., \& Cuevas, S. 1995, J. Opt. Soc. Am. A, 12, 2523

Weigelt, G. P. 1977, Opt. Comm., 21, 55

Wiehr, E., Bovelet, B., \& Hirzberger, J. 2004, A\&A, 422, L63

Winker, D. M. 1991, J. Opt. Soc. Am. A, 8, 1568

Wunnenberg, M., Kneer, F., \& Hirzberger, J. 2002, A\&A, 395, L51 